\title{
Determining the extent of biodegradation of fuels using the diastereomers of the acyclic isoprenoids
}

\author{
Cameron P. McIntyre *ף, Paul McA. Harvey ${ }^{*}$, Susan H. Ferguson ${ }^{*}$, Anna M. \\ Wressnig ${ }^{\S}$, Herbert Volk ${ }^{\dagger}$, Simon C. George ${ }^{¥}$ and Ian Snape ${ }^{\dot{*}}$ \\ ${ }^{\dagger}$ CSIRO Petroleum, PO Box 136, North Ryde, NSW 1670, Australia.
}

" Australia and College of Health and Science, University of Western Sydney, Locked Bag 1797, Penrith South DC 1797, Australia.

${ }^{\ddagger}$ Australian Antarctic Division, Channel Hwy, Kingston, TAS 7050, Australia.

${ }^{\S}$ Institute for Water and Environmental Resource Management, Department of Environmental Sciences, University of Technology, Broadway, PO Box 123, NSW 2007, Australia.

$¥$ Australian Centre for Astrobiology, Macquarie University, North Ryde, NSW 2109, Australia.

* Corresponding Author: email: cpm@cpmcintyre.com; phone 6129490 8245; fax 61294908921.

\section{Supporting Information}

3 tables, 2 figures and 1 text object. 
Table S1.

The biodegradation scale for the determination of the biodegradation level of crude oils.

\begin{tabular}{|c|c|c|}
\hline $\begin{array}{l}\text { Level of } \\
\text { Biodegradation }\end{array}$ & Aliphatic Hydrocarbons & Aromatic Hydrocarbons \\
\hline 1 (Minor) & $\begin{array}{l}\text { Low molecular weight } n \text { - } \\
\text { alkanes depleted }\end{array}$ & MNs depleted \\
\hline 2 & $\begin{array}{l}\text { General depletion of } n \text { - } \\
\text { alkanes }\end{array}$ & DMNs altered \\
\hline 3 & $\begin{array}{l}>90 \% n \text {-alkanes absent, } \\
\text { alkylcyclochexanes affected }\end{array}$ & $\begin{array}{l}\text { DMNs, TMNs, MBPs, EBPs and MDPMs } \\
\text { altered, DMBPs slightly altered }\end{array}$ \\
\hline 4 (Moderate) & $\begin{array}{l}n \text {-Alkanes and } \\
\text { alkylcyclochexanes absent, } \\
\text { acyclic isoprenoids depleted }\end{array}$ & $\begin{array}{l}\text { DMNs, TMNs, MPs altered, MBPs, EBPs, } \\
\text { MDPMs and DMBPs severely depleted, } \\
\text { TMBPs altered }\end{array}$ \\
\hline 5 & $\begin{array}{l}\text { Acyclic isoprenoids severely } \\
\text { depleted }\end{array}$ & $\begin{array}{l}\text { DMNs severely depleted, TMNs, TeMNs } \\
\text { and DMPs altered }\end{array}$ \\
\hline $5-6$ & $\begin{array}{l}\text { Acyclic isoprenoids absent, } \\
\text { bicyclic alkanes affected }\end{array}$ & $\begin{array}{l}\text { DMNs absent, TMNs severely depleted, } \\
\text { TeMNs and DMPs altered, MPs depleted }\end{array}$ \\
\hline 6 (Extensive) & $\begin{array}{l}\text { Bicyclic alkanes severely } \\
\text { depleted, steranes affected, } \\
\text { 25-norhopanes present }\end{array}$ & $\begin{array}{l}\text { TMNs and MPs absent, TeMNs depleted, } \\
\text { DMPs altered, EPs unaltered }\end{array}$ \\
\hline $\begin{array}{l}7 \text { (Very } \\
\text { Extensive) }\end{array}$ & $\begin{array}{l}>50 \% \alpha \alpha \alpha \text { steranes removed, } \\
25 \text {-norhopanes present }\end{array}$ & $\begin{array}{l}\text { DMNs, TMNs, TeMNs, MPs, DMPs, } \\
\text { MBPs, DMBPs, EBPS and MDPMs } \\
\text { absent, EPs and TMBPs severely } \\
\text { depleted }\end{array}$ \\
\hline 8 (Severe) & $\begin{array}{l}\text { Hopanes affected, steranes } \\
\text { absent, diamondoids affected }\end{array}$ & \\
\hline 9 & $\begin{array}{l}\text { Hopanes absent, diasteranes } \\
\text { affected }\end{array}$ & \\
\hline 10 (Extreme) & $\begin{array}{l}\text { Diamondoids severely } \\
\text { affected }\end{array}$ & Aromatic steroids affected \\
\hline
\end{tabular}

$\mathrm{MN}=$ methylnaphthalene, $\mathrm{DMN}=$ dimethylnaphthalene, $\mathrm{TMN}=$ trimethylnaphthalene, $\mathrm{TeMN}=$ tetramethylnaphthalene, $\mathrm{MP}=$ methylphenanthrene, $\mathrm{DMP}=$ dimethylphenanthrene, $\mathrm{EP}=$ ethylphenanthrene, MBP = methylbiphenyl, DMBP = dimethylbiphenyl, TMBP = trimethylbiphenyl, $\mathrm{EBP}=$ ethylbiphenyl, MDPM = methyldiphenylmethane. Adapted from references 1-6. 


\section{Text S1. Site, remediation trial, sample and GC-FID analysis details (24).}

The study site is located in East Antarctica near the Old Casey Research Station. Approximately $1000 \mathrm{~m}^{3}$ of soil is contaminated from historical fuel spills that occurred over 20 years ago. Total petroleum hydrocarbons (TPH) concentrations at the site range between 10,000 and 47,000 mg kg-1. The main contaminant is Special Antarctic Blend (SAB) diesel, which contains predominantly n-alkanes. A land farming trial was initiated in 1998 and has been previously described (25). Briefly, contaminated soil was sieved $(<5.0 \mathrm{~mm})$ and homogenized before being added to hexane-cleaned metal containers (240 (w) x 250 (l) x 150 (d) $\mathrm{mm}$ ) with perforated bottoms. The soil added to the containers had approximately $23,000 \mathrm{mg}$ fuel $\mathrm{kg}^{-1}$ soil. This has currently been revised down to 16,000 $20,000 \mathrm{mg}$ fuel $\mathrm{kg}^{-1}$ following a reassessment of the early analytical procedure for TPH. There were substantial evaporative losses in the first 3 weeks with the fuel concentration measured as $14,300 \mathrm{mg}$ fuel kg${ }^{-1}$ soil after 3 weeks. The containers were variously amended and reburied adjacent to the contaminated site so that environmental conditions were similar to the main plume. Soil temperatures at the site have been measured during summer as between -4 and $23{ }^{\circ} \mathrm{C}$ (4 Dec 2000 to 10 am $27 \mathrm{Feb} 2001$ ). Three treatments are considered here: a fertilization trial in which controlled release fertilizer pellets $\operatorname{MaxBac}{ }^{\circledR}$ were added to the soil to yield soil concentrations of $10 \mathrm{gm}^{-3}$ and $35 \mathrm{gm}^{-3}$ on a dry mass basis; and a control treatment that received no additional nutrients.

The containers were sampled at the top $(0-35 \mathrm{~mm})$, middle $(35-70 \mathrm{~mm})$, and bottom $(70$ $100 \mathrm{~mm}$ ) after 60 months of incubation, and duplicate samples were stored at $-18{ }^{\circ} \mathrm{C}$ in metal tins and glass containers. Sub-samples of soil $(10 \mathrm{~g})$ were weighed into $40 \mathrm{~mL}$ glass vials and spiked with an internal standard $\left(1000 \mathrm{mg} \mathrm{L}^{-1}\right.$ Cyclooctane and 1Bromoeicosane and $100 \mathrm{mg} \mathrm{L}^{-1}$ Naphthalene-d8 and p-Terphenyl). Water (10mL) and hexane $(10 \mathrm{~mL})$ were added, and the samples were tumbled overnight. Analysis of the hexane layer was undertaken on an Agilent $6890 \mathrm{GC}$ fitted with a split/splitless injector $\left(280^{\circ} \mathrm{C}\right)$ and a flame ionization detector (FID) $\left(300^{\circ} \mathrm{C}\right)$. Separation was performed on a $50 \mathrm{~m} \times 0.22 \mathrm{~mm}$ i.d. $\times 0.25 \mu \mathrm{m}$ film BP-1 (SGE) column with helium as the carrier gas. The $\mathrm{GC}$ oven program was $40{ }^{\circ} \mathrm{C}$ for $5 \mathrm{~min}$, and then ramped to $310{ }^{\circ} \mathrm{C}\left(\right.$ at $10^{\circ} \mathrm{C} \mathrm{min}^{-1}$ ) and held for $15 \mathrm{~min}$. The instrument was cross calibrated with an in-house SAB standard. The quantity of fuel was approximated by the sum of resolved peaks and the undefined complex mixture between $\mathrm{C}_{9}$ and $\mathrm{C}_{28}$ and normalized to the amount of internal standard recovered. 
Table S2. Compounds and SIM parameters used.

\begin{tabular}{|c|c|c|c|c|c|c|}
\hline Compound name & Initialism & $\begin{array}{l}\text { Rentention time } \\
\text { (min. approx.) }\end{array}$ & $\begin{array}{l}m / z \text { used } \\
(\mathrm{Da})\end{array}$ & $\begin{array}{l}\text { Group } \\
\text { number }\end{array}$ & $\begin{array}{l}\text { lons in } \\
\text { group }\end{array}$ & $\begin{array}{l}\text { Dwell time } \\
\text { (ms) }\end{array}$ \\
\hline \multicolumn{7}{|l|}{ Internal Standards } \\
\hline Cyclooctane & - & 10.62 & 112 & 1 & 7 & 25 \\
\hline Naphthalene-d8 & - & 15.13 & 136 & 5 & 9 & 25 \\
\hline Anthracene-d10 & - & 23.07 & 188 & 11 & 6 & 25 \\
\hline p-Terphenyl & - & 26.86 & 230 & 11 & 6 & 25 \\
\hline 1-Bromoeicosane & - & 28.15 & 135 & 11 & 6 & 25 \\
\hline \multicolumn{7}{|l|}{ n-Alkanes } \\
\hline Octane & $n \mathrm{C} 8$ & 8.22 & 57 & 1 & 7 & 25 \\
\hline Nonane & $n \mathrm{C9}$ & 10.08 & 57 & 1 & 7 & 25 \\
\hline Decane & $n \mathrm{C} 10$ & 11.87 & 57 & 1 & 7 & 25 \\
\hline Undecane & $n \mathrm{C} 11$ & 13.53 & 57 & 2 & 6 & 25 \\
\hline Dodecane & $n \mathrm{C} 12$ & 15.09 & 57 & 5 & 9 & 25 \\
\hline Tridecane & $n \mathrm{C} 13$ & 16.54 & 57 & 7 & 6 & 25 \\
\hline Tetradecane & $n \mathrm{C} 14$ & 17.90 & 57 & 8 & 6 & 25 \\
\hline Pentadecane & $n \mathrm{C} 15$ & 19.18 & 57 & 9 & 6 & 25 \\
\hline Hexadecane & $n \mathrm{C} 16$ & 20.38 & 57 & 10 & 6 & 25 \\
\hline Heptadecane & $n \mathrm{C} 17$ & 21.52 & 57 & 10 & 6 & 25 \\
\hline Octadecane & $n \mathrm{C} 18$ & 22.60 & 57 & 10 & 6 & 25 \\
\hline Nonadecane & $n C 19$ & 23.63 & 57 & 11 & 6 & 25 \\
\hline Eicosane & $n \mathrm{C} 20$ & 24.61 & 57 & 11 & 6 & 25 \\
\hline Heneicosane & $n \mathrm{C} 21$ & 25.55 & 57 & 11 & 6 & 25 \\
\hline Docosane & $n \mathrm{C} 22$ & 26.44 & 57 & 11 & 6 & 25 \\
\hline Tricosane & $n \mathrm{C} 23$ & 27.30 & 57 & 11 & 6 & 25 \\
\hline Pentacosane & $n \mathrm{C} 24$ & 28.91 & 57 & 11 & 6 & 25 \\
\hline Hexacosane & $n \mathrm{C} 25$ & 29.68 & 57 & 11 & 6 & 25 \\
\hline Heptacosane & $n \mathrm{C} 26$ & 30.42 & 57 & 11 & 6 & 25 \\
\hline Octacosane & $n \mathrm{C} 27$ & 31.18 & 57 & 11 & 6 & 25 \\
\hline Nonacosane & $n \mathrm{C} 28$ & 32.00 & 57 & 11 & 6 & 25 \\
\hline Triacontane & $n \mathrm{C} 29$ & 32.88 & 57 & 11 & 6 & 25 \\
\hline Eitriacontane & $n \mathrm{C} 30$ & 33.87 & 57 & 12 & 2 & 25 \\
\hline Dotriacontane & $n \mathrm{C} 31$ & 34.98 & 57 & 12 & 2 & 25 \\
\hline Tritriacontane & $n \mathrm{C} 32$ & 36.29 & 57 & 12 & 2 & 25 \\
\hline \multicolumn{7}{|l|}{ Acyclic Isoprenoids } \\
\hline $\mathrm{C}_{13}$-isoprenoid & $i \mathrm{C} 13$ & 15.31 & 57 & 5 & 9 & 25 \\
\hline $\mathrm{C}_{14}$-isoprenoid & $i \mathrm{C} 14$ & 16.16 & 57 & 6 & 6 & 25 \\
\hline $\mathrm{C}_{15}$-isoprenoid & $i C 15$ & 17.61 & 57 & 8 & 6 & 25 \\
\hline $\mathrm{C}_{16}$-isoprenoid & $i C 16$ & 18.71 & 57 & 8 & 6 & 25 \\
\hline Norpristane & $\mathrm{nPr}$ & 20.95 & 57 & 10 & 6 & 25 \\
\hline Pristane & $\mathrm{Pr}$ & 21.60 & 57 & 10 & 6 & 25 \\
\hline Phytane & $\mathrm{Ph}$ & 22.72 & 57 & 10 & 6 & 25 \\
\hline \multicolumn{7}{|l|}{ Alkylcyclohexanes } \\
\hline Cyclohexane, propyl- & $\mathrm{PCH}$ & 10.75 & 83 & 1 & 7 & 25 \\
\hline Cyclohexane, butyl- & $\mathrm{BCH}$ & 12.55 & 83 & 1 & 7 & 25 \\
\hline Cyclohexane, pentyl- & $\mathrm{PeCH}$ & 14.24 & 83 & 4 & 7 & 25 \\
\hline Cyclohexane, hexyl- & $\mathrm{CH} 6$ & 15.82 & 83 & 6 & 6 & 25 \\
\hline Cyclohexane, heptyl- & $\mathrm{CH} 7$ & 17.29 & 83 & 7 & 6 & 25 \\
\hline Cyclohexane, octyl- & $\mathrm{CH} 8$ & 18.67 & 83 & 8 & 6 & 25 \\
\hline Cyclohexane, nonyl- & $\mathrm{CH} 9$ & 19.97 & 83 & 10 & 6 & 25 \\
\hline Cyclohexane, decyl- & $\mathrm{CH} 10$ & 21.18 & 83 & 10 & 6 & 25 \\
\hline Cyclohexane, undecyl- & $\mathrm{CH} 11$ & 22.34 & 83 & 10 & 6 & 25 \\
\hline Cyclohexane, dodecyl- & $\mathrm{CH} 12$ & 23.42 & 83 & 11 & 6 & 25 \\
\hline Cyclohexane, tridecyl- & $\mathrm{CH} 13$ & 24.46 & 83 & 11 & 6 & 25 \\
\hline Cyclohexane, tetradecyl- & $\mathrm{CH} 14$ & 25.44 & 83 & 11 & 6 & 25 \\
\hline Cyclohexane, pentadecyl- & $\mathrm{CH} 15$ & 26.39 & 83 & 11 & 6 & 25 \\
\hline \multicolumn{7}{|l|}{ Methylalkylcyclohexanes } \\
\hline Cyclohexane, trans -1-methyl-4-ethyl- & $\mathrm{t} 14 \mathrm{EMCH}$ & 9.96 & 97 & 1 & 7 & 25 \\
\hline Cyclohexane, methyl-propyl- & $\mathrm{MPCH}$ & 11.69 & 97 & 1 & 7 & 25 \\
\hline Cyclohexane, methyl-butyl- & $\mathrm{MBCH}$ & 13.41 & 97 & 2 & 6 & 25 \\
\hline Cyclohexane, methyl-pentyl- & $\mathrm{MPeCH}$ & 15.02 & 97 & 5 & 9 & 25 \\
\hline
\end{tabular}


Cyclohexane, methyl-hexyl-

Cyclohexane, methyl-heptyl-

Cyclohexane, methyl-octyl-

Cyclohexane, methyl-nonyl-

$\mathrm{MCH} 1 / 6$

$\mathrm{MCH} 1 / 7$

$\mathrm{MCH} 1 / 9$

19.27
20.51

Bicyclic terpanes

$\mathrm{C}_{15}$-bicyclic sequiterpane

$\mathrm{C}_{15}$-bicyclic sequiterpane

b15g

18.66

Drimane

Homodrimane

Decahydronaphthalene

Decahydronaphthalene, 2-methyl-

Decahydronaphthalene, 1-methyl-

Spiroundecane

Decahydronaphthalene, dimethyl-

Decahydronaphthalene, dimethyl-

Decahydronaphthalene, dimethyl-

Decahydronaphthalene, dimethyl-

Bicyclohexane

b15k

19.15

DHN

MDHN 13.99

MDHN $\quad 14.28$

SU $\quad 15.09$

DMDHN-a

DMDHN-b $\quad 15.13$

DMDHN-c $\quad 15.77$

DMDHN-d $\quad 15.79$

$\mathrm{BICH} \quad 16.97$

\section{Hopanes}

$\mathrm{C}_{27}$ 18a(H)-22,29,30-trisnorneohopane

$\mathrm{C}_{27}$ 17 $\mathrm{\alpha}(\mathrm{H})-22,29,30$-trisnorhopane

$17 \alpha(\mathrm{H}), 21 \beta(\mathrm{H})-30$-norhopane

18 $\alpha(\mathrm{H})$-30-norneohopane

$17 \alpha(H), 21 \beta(H)$-hopane

$17 \alpha(H), 21 \beta(H)-30$-homohopane (22S)

$17 \alpha(\mathrm{H}), 21 \beta(\mathrm{H})-30$-homohopane $(22 R)$

$17 \alpha(\mathrm{H}), 21 \beta(\mathrm{H})$-30-bishomohopane (22S)

$17 \alpha(H), 21 \beta(H)-30$-bishomohopane (22R)

Ts

33.39

Tm 33.63

C29a 34.81

C29Ts $\quad 34.85$

C30 $\mathrm{\alpha} \quad 35.79$

C31 $\alpha$ 22S $\quad 37.14$

C31 $\alpha$ 22R

C32 $\alpha$ B22S $\quad 38.31$

C32 $\alpha 322 \mathrm{R} \quad 38.62$

Adamantanes

Adamantane

1-Methyladamantane

1,3-Dimethyladamantane

1,3,5-Trimethyladamantane

1,3,5,7-Tetramethyladamantane

2-Methyladamantane

cis -1,4-Dimethyladamantane

trans -1,4-Dimethyladamantane

1,3,6-Trimethyladamantane

1,2-Dimethyladamantane

cis -1,3,4-Trimethyladamantane

trans -1,3,4-Trimethyladamantane

1,2,5,7-Tetramethyladamantane

A

1-MA

13.62

14.13

14.28

1357-TeMA $\quad 14.40$

2-MA $\quad 14.96$

14c-DMA $\quad 15.10$

14t-DMA $\quad 15.19$

136-TMA $\quad 15.27$

12-DMA $\quad 15.63$

134c-TMA $\quad 15.71$

134t-TMA 15.78

1257-TeMA $\quad 15.81$

C3 and C4 Benzenes

Benzene, Isopropyl-

Benzene, $n$-Propyl-

Benzene, 1-methyl-3-ethyl-

Benzene, 1-methyl-4-ethyl-

Benzene, 1,3,5-trimethyl

Benzene, 1-methyl-2-ethyl-

Benzene, 1,2,4-trimethyl

Benzene, 1,2,3-trimethyl

Benzene, Isobutyl-

Benzene, Secbutyl-

Benzene, 1-methyl-3-isopropyl-

Benzene, 1-methyl-4-isopropyl-

Benzene, 1-methyl-2-isopropyl-

Benzene, 1,3-diethyl-

Benzene, 1-methyl-3-propyl-

Benzene, 1-methyl-4-propyl-

Benzene, 1,4-diethyl-

Benzene, $n$-Butyl-

Benzene, 1,2-diethyl-

IPB

NPB

11.33

35TMB $\quad 11.42$

$1 \mathrm{M} 2 \mathrm{~EB} \quad 11.65$

124TMB $\quad 11.89$

12.42

BB 12.14

$\begin{array}{ll}\text { SBB } & 12.19\end{array}$

M3IPB $\quad 12.35$

$\begin{array}{ll}M 4 I P B & 12.41\end{array}$

M2IPB $\quad 12.67$

13DEB $12.86,12.97$

co-elutes

1M4PB co-elutes

14DEB co-elutes

nBB co-elutes

12DEB co-elutes

$\begin{array}{llll}97 & 7 & 6 & 25 \\ 97 & 8 & 6 & 25 \\ 97 & 9 & 6 & 25 \\ 97 & 10 & 6 & 25\end{array}$

$\begin{array}{llll}193 & 8 & 6 & 25 \\ 193 & 9 & 6 & 25 \\ 123 & 9 & 6 & 25 \\ 123 & 10 & 6 & 25 \\ 138 & 2 & 6 & 25 \\ 152 & 3 & 6 & 25 \\ 152 & 4 & 7 & 25 \\ 152 & 5 & 9 & 25 \\ 166 & 5 & 9 & 25 \\ 166 & 5 & 9 & 25 \\ 166 & 6 & 6 & 25 \\ 166 & 6 & 6 & 25 \\ 166 & 7 & 6 & 25\end{array}$

$\begin{array}{llll}191 & 12 & 2 & 200 \\ 191 & 12 & 2 & 200 \\ 191 & 12 & 2 & 200 \\ 191 & 12 & 2 & 200 \\ 191 & 12 & 2 & 200 \\ 191 & 12 & 2 & 200 \\ 191 & 12 & 2 & 200 \\ 191 & 12 & 2 & 200 \\ 191 & 12 & 2 & 200\end{array}$

$\begin{array}{llll}136 & 2 & 6 & 25 \\ 135 & 3 & 6 & 25 \\ 149 & 3 & 6 & 25 \\ 163 & 4 & 7 & 25 \\ 177 & 4 & 7 & 25 \\ 135 & 5 & 9 & 25 \\ 149 & 5 & 9 & 25 \\ 149 & 5 & 9 & 25 \\ 163 & 5 & 9 & 25 \\ 149 & 6 & 6 & 25 \\ 163 & 6 & 6 & 25 \\ 163 & 6 & 6 & 25 \\ 177 & 6 & 6 & 25\end{array}$

$\begin{array}{llll}120 & 1 & 7 & 25 \\ 120 & 1 & 7 & 25 \\ 120 & 1 & 7 & 25 \\ 120 & 1 & 7 & 25 \\ 120 & 1 & 7 & 25 \\ 120 & 1 & 7 & 25 \\ 120 & 1 & 7 & 25 \\ 120 & 1 & 7 & 25 \\ 134 & 1 & 7 & 25 \\ 134 & 1 & 7 & 25 \\ 134 & 1 & 7 & 25 \\ 134 & 1 & 7 & 25 \\ 134 & 1 & 7 & 25 \\ 134 & 1 & 7 & 25 \\ 134 & 1 & 7 & 25 \\ 134 & 1 & 7 & 25 \\ 134 & 1 & 7 & 25 \\ 134 & 1 & 7 & 25 \\ 134 & 1 & 7 & 25\end{array}$


Benzene, 1,3-dimethyl-5-ethylBenzene, 1-methyl-2-propyl-

Benzene, 1,4-dimethyl-2-ethylBenzene, 1,3-dimethyl-4-ethylBenzene, 1,2-dimethyl-4-ethylBenzene, 1,3-dimethyl-2-ethylBenzene, 1,2-dimethyl-3-ethylBenzene, 1,2,4,5-tetramethyl Benzene, 1,2,3,5-tetramethy

Benzene, 1,2,3,4-tetramethyl

C0-C4 Naphthalenes

Naphthalene

Naphthalene, 2-methyl-

Naphthalene, 1-methyl-

Naphthalene, 1-ethyl-

Naphthalene, 2-ethyl-

Naphthalene, 2,6-dimethyl-

Naphthalene, 2,7-dimethyl-

Naphthalene, 1,3-dimethyl-

Naphthalene, 1,7-dimethyl-

Naphthalene, 1,6-dimethyl-

Naphthalene, 1,4-dimethylNaphthalene, 2,3-dimethyl-

Naphthalene, 1,5-dimethyl-

Naphthalene, 1,2-dimethyl-

Ethylmethylnaphthlenes

Naphthalene, 1,3,7-trimethyl-

Naphthalene, 1,3,6-trimethy-

Naphthalene, 1,3,5-trimethyl-

Naphthalene, 1,4,6-trimethyl-

Naphthalene, 2,3,6-trimethyl-

Naphthalene, 1,2,7-trimethyl-

Naphthalene, 1,6,7-trimethyl-

Naphthalene, 1,2,6-trimethyl-

Naphthalene, 1,2,4-trimethyl-

Naphthalene, 1,2,5-trimethyl-

Naphthalene, 1,2,3-trimethyl-

Naphthalene, 1,3,6,7-tetramethyl-

Naphthalene, 1,2,4,6-tetramethyl-

Naphthalene, 1,2,4,7-tetramethyl-

Naphthalene, 1,4,6,7-tetramethyl-

Naphthalene, 1,2,5,7-tetramethyl-

Naphthalene, 2,3,6,7-tetramethyl-

Naphthalene, 1,2,6,7-tetramethyl-

Naphthalene, 1,2,3,7-tetramethyl-

Naphthalene, 1,2,3,6-tetramethyl-

Naphthalene, 1,2,5,6-tetramethyl-

Naphthalene, 1,2,3,5-tetramethyl-

C0 and C1 Biphenyls

Biphenyl

3-Methylbiphenyl

4-Methylbiphenyl

CO and C1 Phenanthrenes

Phenanthrene

3-Methylphenanthrene

2-Methylphenanthrene

9-Methylphenanthrene

1-Methylphenanthrene

Monoaromatic bicyclics

Indane

Methylindane

Tetralin

Dimethylindane

Tetralin, methyl-

Tetralin, methyl-

$\begin{array}{llllll}\text { 13D5EB } & \text { co-elutes } & 134 & 1 & 7 & 25 \\ \text { 1M2PB } & 13.16 & 134 & 2 & 6 & 25 \\ \text { 14D2EB } & 13.31 & 134 & 2 & 6 & 25 \\ \text { 13D4EB } & 13.35 & 134 & 2 & 6 & 25 \\ \text { 12D4EB } & 13.45 & 134 & 2 & 6 & 25 \\ \text { 13D2EB } & 13.58 & 134 & 2 & 6 & 25 \\ \text { 12D3EB } & 13.83 & 134 & 3 & 6 & 25 \\ \text { 1245TeMB } & 13.97 & 134 & 3 & 6 & 25 \\ \text { 1235TeMB } & 14.05 & 134 & 3 & 6 & 25 \\ \text { 1234TeMB } & 14.59 & 134 & 4 & 7 & 25\end{array}$

$\mathrm{N}$

2-MN 15.18

2-EN

co-elutes

co-elutes
18.54

co-elutes

18.59

18.88

co-elutes

18.88

19.05

19.62

19.89

19.96

20.16

co-elutes

20.21

20.38

co-elutes

20.42

20.62

20.71

20.94

21.66

21.82

co-elutes

co-elutes

21.87

21.95

22.07

22.13

22.20

22.40

co-elutes

1256-TeMN

1235-TeMN

17.96

3-MBp

4-MBp

19.31

19.45

23.02

3-MP

24.19

24.27

2-MP

9-MP

24.53

13.53

14.58

14.80

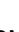

16.31

16.35

16.77

142

156

156

56

156

156

56

156
156

156

156

170

170

70

170
170

170

170

170

170

170

170

170
184

184

184

184

184
184

184

184

184

$\begin{array}{llll}154 & 8 & 6 & 25 \\ 168 & 9 & 6 & 25 \\ 168 & 9 & 6 & 25\end{array}$

Mtet-b

178

192

192

192

$\begin{array}{lll}11 & 6 & 25 \\ 11 & 6 & 25 \\ 11 & 6 & 25 \\ 11 & 6 & 25 \\ 11 & 6 & 25\end{array}$

$\begin{array}{llll}117 & 2 & 6 & 25 \\ 132 & 4 & 7 & 25 \\ 132 & 4 & 7 & 25 \\ 146 & 7 & 6 & 25 \\ 146 & 7 & 6 & 25 \\ 146 & 7 & 6 & 25\end{array}$


Table S3

Analysis results of soils contaminated with petroleum hydrocarbons taken from a bioremediation trial at Casey Station, Antarctica.

\begin{tabular}{|c|c|c|c|c|c|c|c|c|c|c|c|c|}
\hline Tin & Sample & Type & Depth & 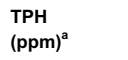 & $\begin{array}{l}n \mathrm{C} 17 \\
\text { / Pristane }^{\mathrm{a}}\end{array}$ & $\begin{array}{l}n \text { C18 } \\
\text { / Phytane }\end{array}$ & $\begin{array}{l}\text { Pristane / } \\
\text { Phytane }^{\text {a }}\end{array}$ & $\begin{array}{l}\text { Pris+Phyt } \\
{\text { I } \sum \text { Hopanes }^{\mathrm{b}}}\end{array}$ & $\mathrm{BDL}^{\mathrm{a}}$ & $\begin{array}{l}\text { nPrDR } \\
( \pm 0.01)^{b}\end{array}$ & $\begin{array}{l}\text { PrDR } \\
( \pm 0.01)^{b}\end{array}$ & $\begin{array}{l}\mathrm{PhDR}^{*} \\
( \pm 0.01)^{\mathrm{b}}\end{array}$ \\
\hline 1 & 339 & CTRL & Top & $4,080 \pm 230$ & $1.38 \pm 0.29$ & $0.29 \pm 0.01$ & $8.1 \pm 0.3$ & 8.5 & 1 & 1.02 & 1.10 & 0.94 \\
\hline 1 & 340 & CTRL & Top & $5,540 \pm 260$ & $1.48 \pm 0.30$ & $0.30 \pm 0.02$ & $8.6 \pm 0.4$ & 9.5 & 1 & 1.00 & 1.06 & 0.95 \\
\hline 1 & 341 & CTRL & Mid & $8,430 \pm 320$ & $1.04 \pm 0.20$ & $0.20 \pm 0.01$ & $9.5 \pm 0.4$ & 12.4 & 1 & 0.97 & 1.08 & 0.90 \\
\hline 1 & 342 & CTRL & Mid & $10,930 \pm 380$ & $1.00 \pm 0.19$ & $0.19 \pm 0.01$ & $10 \pm 0.6$ & 12.4 & 1 & 0.97 & 1.08 & 0.90 \\
\hline 1 & 343 & CTRL & Base & $10,980 \pm 390$ & $0.95 \pm 0.18$ & $0.18 \pm 0.01$ & $9.8 \pm 0.4$ & 11.8 & 1 & 0.97 & 1.07 & 0.92 \\
\hline 1 & 344 & CTRL & Base & $9,540 \pm 350$ & $0.96 \pm 0.18$ & $0.18 \pm 0.01$ & $9.8 \pm 0.5$ & 12.2 & 1 & 0.98 & 1.05 & 0.90 \\
\hline 2 & 327 & CTRL & Top & $6,670 \pm 280$ & $1.26 \pm 0.25$ & $0.25 \pm 0.01$ & $8.6 \pm 0.4$ & 9.0 & 1 & 1.01 & 1.06 & 0.96 \\
\hline 2 & 328 & CTRL & Top & $7,160 \pm 290$ & $1.27 \pm 0.25$ & $0.25 \pm 0.01$ & $9 \pm 0.4$ & 10.0 & 1 & 0.98 & 1.07 & 0.93 \\
\hline 2 & 329 & CTRL & Mid & $10,030 \pm 360$ & $0.98 \pm 0.19$ & $0.19 \pm 0.01$ & $9.7 \pm 0.5$ & 10.4 & 1 & 0.98 & 1.04 & 0.93 \\
\hline 2 & 330 & CTRL & Mid & $11,070 \pm 390$ & $0.96 \pm 0.18$ & $0.18 \pm 0.01$ & $9.8 \pm 0.5$ & 11.9 & 1 & 0.99 & 1.08 & 0.92 \\
\hline 2 & 331 & CTRL & Base & $11,000 \pm 390$ & $0.96 \pm 0.18$ & $0.18 \pm 0.01$ & $9.9 \pm 0.8$ & 10.2 & 1 & 0.99 & 1.06 & 0.92 \\
\hline 2 & 332 & CTRL & Base & $11,490 \pm 400$ & $0.97 \pm 0.18$ & $0.18 \pm 0.01$ & $10 \pm 0.5$ & 10.4 & 1 & 0.98 & 1.08 & 0.92 \\
\hline 3 & 422 & CTRL & Top & $6,380 \pm 280$ & $1.22 \pm 0.24$ & $0.24 \pm 0.01$ & $9 \pm 0.4$ & 9.2 & 1 & 0.97 & 1.06 & 0.95 \\
\hline 3 & 423 & CTRL & Top & $4,730 \pm 250$ & $1.29 \pm 0.26$ & $0.26 \pm 0.01$ & $8.4 \pm 0.5$ & 8.1 & 1 & 0.99 & 1.06 & 0.93 \\
\hline 3 & 424 & CTRL & Mid & $12,650 \pm 430$ & $0.96 \pm 0.19$ & $0.19 \pm 0.01$ & $9.8 \pm 0.5$ & 11.8 & 1 & 0.96 & 1.08 & 0.92 \\
\hline 3 & 425 & CTRL & Mid & $10,360 \pm 370$ & $0.98 \pm 0.18$ & $0.18 \pm 0.01$ & $9.9 \pm 0.6$ & 11.5 & 1 & 0.97 & 1.05 & 0.93 \\
\hline 3 & 426 & CTRL & Base & $14,090 \pm 470$ & $0.94 \pm 0.18$ & $0.18 \pm 0.01$ & $9.8 \pm 0.5$ & 11.8 & 1 & 0.99 & 1.07 & 0.92 \\
\hline 3 & 427 & CTRL & Base & $13,670 \pm 460$ & $0.92 \pm 0.18$ & $0.18 \pm 0.01$ & $9.9 \pm 0.5$ & 12.3 & 1 & 0.96 & 1.07 & 0.94 \\
\hline 4 & 381 & CTRL & Top & $5,330 \pm 260$ & $1.30 \pm 0.25$ & $0.25 \pm 0.01$ & $9.1 \pm 0.5$ & 9.7 & 1 & 1.02 & 1.06 & 0.93 \\
\hline 4 & 382 & CTRL & Top & $4,220 \pm 240$ & $1.52 \pm 0.31$ & $0.31 \pm 0.02$ & $8.4 \pm 0.5$ & 8.5 & 1 & 0.98 & 1.05 & 0.91 \\
\hline 4 & 383 & CTRL & Mid & $9,760 \pm 350$ & $1.01 \pm 0.20$ & $0.20 \pm 0.01$ & $9.6 \pm 0.5$ & 11.7 & 1 & 0.98 & 1.08 & 0.91 \\
\hline 4 & 384 & CTRL & Mid & $9,750 \pm 350$ & $1.00 \pm 0.20$ & $0.20 \pm 0.01$ & $9.6 \pm 0.5$ & 10.6 & 1 & 1.00 & 1.04 & 0.92 \\
\hline 4 & 385 & CTRL & Base & $8,790 \pm 330$ & $0.95 \pm 0.19$ & $0.19 \pm 0.01$ & $9.5 \pm 0.4$ & 10.3 & 1 & 0.94 & 1.05 & 0.92 \\
\hline 4 & 386 & CTRL & Base & $11,310 \pm 390$ & $0.96 \pm 0.18$ & $0.18 \pm 0.01$ & $10 \pm 0.5$ & 11.7 & 1 & 0.98 & 1.08 & 0.93 \\
\hline 5 & 428 & HCRN & Top & $2,040 \pm 210$ & - & - & $4.2 \pm 0.5$ & 1.2 & 5 & 0.66 & 0.61 & 0.67 \\
\hline 5 & 429 & HCRN & Top & $4,310 \pm 240$ & - & - & $7 \pm 0.4$ & 4.3 & 4 & 0.57 & 0.79 & 0.88 \\
\hline 5 & 430 & HCRN & Mid & $5,930 \pm 270$ & - & - & $7.7 \pm 0.4$ & 4.8 & 4 & 0.69 & 0.83 & 0.89 \\
\hline 5 & 431 & HCRN & Mid & $4,970 \pm 250$ & - & - & $7.9 \pm 0.4$ & 4.6 & 4 & 0.78 & 0.87 & 0.90 \\
\hline 5 & 432 & HCRN & Base & $5,570 \pm 260$ & - & - & $7.6 \pm 0.5$ & 4.3 & 4 & 0.67 & 0.79 & 0.86 \\
\hline 5 & 433 & HCRN & Base & $6,200 \pm 270$ & - & - & $6.4 \pm 0.5$ & 2.9 & 5 & 0.61 & 0.68 & 0.74 \\
\hline 6 & 369 & HCRN & Top & $3,910 \pm 230$ & - & - & $4.4 \pm 1.4$ & 0.6 & 5 & 0.52 & 0.48 & 0.58 \\
\hline 6 & 370 & HCRN & Top & $570 \pm 200$ & - & - & $2.9 \pm 1.1$ & 0.2 & 5 & 0.72 & 0.74 & 0.82 \\
\hline 6 & 371 & HCRN & Mid & $3,850 \pm 230$ & - & - & $6.9 \pm 0.9$ & 1.6 & 4 & 0.72 & 0.81 & 0.84 \\
\hline 6 & 372 & HCRN & Mid & $4,020 \pm 230$ & - & - & $5.4 \pm 1.1$ & 1.6 & 5 & 0.75 & 0.81 & 0.77 \\
\hline 6 & 373 & HCRN & Base & $5,520 \pm 260$ & - & - & $1 \pm 7.1$ & 0.1 & 5 & 0.64 & 0.67 & 0.62 \\
\hline 6 & 374 & HCRN & Base & $5,880 \pm 270$ & - & - & $5.7 \pm 2.3$ & 0.5 & 5 & 0.80 & 0.59 & 0.61 \\
\hline 7 & 410 & HCRN & Top & $1,070 \pm 200$ & - & - & $6.8 \pm 0.3$ & 3.1 & 4 & 0.92 & 0.96 & 0.98 \\
\hline 7 & 411 & HCRN & Top & $970 \pm 200$ & - & - & $5.5 \pm 0.5$ & 1.6 & 5 & 0.91 & 0.91 & 0.95 \\
\hline 7 & 412 & HCRN & Mid & $2,750 \pm 220$ & - & - & $6.4 \pm 0.5$ & 2.8 & 4 & 0.71 & 0.77 & 0.84 \\
\hline 7 & 413 & HCRN & Mid & $6,400 \pm 280$ & - & - & $9.6 \pm 0.4$ & 10.8 & 3 & 0.87 & 1.03 & 1.01 \\
\hline 7 & 414 & HCRN & Base & $5,180 \pm 250$ & - & - & $7.8 \pm 0.6$ & 3.8 & 4 & 0.60 & 0.80 & 0.85 \\
\hline 7 & 415 & HCRN & Base & $4,480 \pm 240$ & - & - & $5.6 \pm 0.7$ & 2.0 & 5 & 0.69 & 0.57 & 0.69 \\
\hline 8 & 357 & HCRN & Top & $1,440 \pm 200$ & - & - & $7 \pm 0.2$ & 5.7 & 4 & 0.84 & 0.95 & 1.02 \\
\hline 8 & 358 & HCRN & Top & $1,730 \pm 210$ & - & - & $6.7 \pm 0.2$ & 4.8 & 4 & 0.77 & 0.90 & 1.03 \\
\hline 8 & 359 & HCRN & Mid & $5,600 \pm 260$ & - & - & $9.6 \pm 0.3$ & 11.0 & 3 & 0.88 & 1.01 & 1.00 \\
\hline 8 & 360 & HCRN & Mid & $5,200 \pm 250$ & - & - & $8.7 \pm 0.3$ & 8.5 & 3 & 0.85 & 0.99 & 0.97 \\
\hline 8 & 361 & HCRN & Base & $5,860 \pm 270$ & - & - & $9.4 \pm 0.3$ & 9.4 & 3 & 0.78 & 0.97 & 1.00 \\
\hline 8 & 362 & HCRN & Base & $5,290 \pm 260$ & - & - & $8.7 \pm 0.3$ & 7.5 & 3 & 0.74 & 0.93 & 0.97 \\
\hline 9 & 363 & LCRN & Top & $3,130 \pm 220$ & - & - & $7.5 \pm 0.3$ & 5.9 & 4 & 0.75 & 0.93 & 0.96 \\
\hline 9 & 364 & LCRN & Top & $1,940 \pm 210$ & - & - & $7.3 \pm 0.3$ & 5.6 & 4 & 0.79 & 0.94 & 0.94 \\
\hline 9 & 365 & LCRN & Mid & $4,480 \pm 240$ & - & - & $8.1 \pm 0.4$ & 6.7 & 4 & 0.67 & 0.88 & 0.95 \\
\hline 9 & 366 & LCRN & Mid & $4,590 \pm 240$ & - & - & $7.3 \pm 0.4$ & 4.7 & 4 & 0.60 & 0.82 & 0.86 \\
\hline 9 & 367 & LCRN & Base & $5,930 \pm 270$ & - & - & $8.2 \pm 0.4$ & 5.3 & 4 & 0.71 & 0.85 & 0.88 \\
\hline 9 & 368 & LCRN & Base & $6,820 \pm 290$ & - & - & $6.4 \pm 0.5$ & 3.5 & 5 & 0.63 & 0.66 & 0.77 \\
\hline 10 & 434 & LCRN & Top & $2,760 \pm 220$ & - & - & $6.9 \pm 0.4$ & 4.8 & 4 & 0.62 & 0.82 & 0.91 \\
\hline 10 & 435 & LCRN & Top & $3,930 \pm 230$ & - & - & $8.1 \pm 0.3$ & 6.9 & 4 & 0.68 & 0.90 & 0.91 \\
\hline 10 & 436 & LCRN & Mid & $2,090 \pm 210$ & - & - & $5.9 \pm 0.3$ & 3.3 & 5 & 0.66 & 0.77 & 0.84 \\
\hline 10 & 437 & LCRN & Mid & $4,840 \pm 250$ & - & - & $8.7 \pm 0.3$ & 8.6 & 3 & 0.74 & 0.89 & 0.94 \\
\hline 10 & 438 & LCRN & Base & $5,880 \pm 270$ & - & - & $9.3 \pm 0.3$ & 8.9 & 3 & 0.74 & 0.95 & 0.97 \\
\hline 10 & 439 & LCRN & Base & $6,330 \pm 280$ & - & - & $9.6 \pm 0.3$ & 9.1 & 3 & 0.76 & 0.96 & 0.99 \\
\hline 101 & 404 & LCRN & Top & $4,480 \pm 240$ & - & - & $9.3 \pm 0.3$ & 9.0 & 3 & 0.89 & 1.03 & 0.98 \\
\hline 101 & 405 & LCRN & Top & $3,500 \pm 230$ & - & - & $8.7 \pm 0.3$ & 8.1 & 3 & 0.84 & 1.00 & 1.01 \\
\hline 101 & 406 & LCRN & Mid & $6,580 \pm 280$ & - & - & $9.4 \pm 0.4$ & 9.0 & 3 & 0.88 & 1.00 & 1.00 \\
\hline 101 & 407 & LCRN & Mid & $5,820 \pm 270$ & - & - & $9.6 \pm 0.4$ & 9.7 & 3 & 0.92 & 1.04 & 1.02 \\
\hline 101 & 408 & LCRN & Base & $7,780 \pm 310$ & - & - & $9.9 \pm 0.4$ & 9.9 & 3 & 0.91 & 1.02 & 1.02 \\
\hline 101 & 409 & LCRN & Base & $7,940 \pm 310$ & - & - & $9.7 \pm 0.3$ & 9.8 & 3 & 0.82 & 1.01 & 0.99 \\
\hline 102 & 352 & LCRN & Top & $3,290 \pm 220$ & - & - & $6.1 \pm 0.6$ & 1.9 & 5 & 0.66 & 0.74 & 0.77 \\
\hline 102 & 353 & LCRN & Top & $6,040 \pm 270$ & - & - & $8.6 \pm 0.3$ & 6.9 & 3 & 0.71 & 0.91 & 0.92 \\
\hline 102 & 354 & LCRN & Mid & $4,990 \pm 250$ & - & - & $5.9 \pm 0.5$ & 2.4 & 5 & 0.60 & 0.63 & 0.74 \\
\hline 102 & 355 & LCRN & Mid & $6,480 \pm 280$ & - & - & $8.8 \pm 0.3$ & 6.9 & 3 & 0.65 & 0.93 & 0.92 \\
\hline 102 & 356 & LCRN & Base & $5,290 \pm 260$ & - & - & $7.6 \pm 0.3$ & 4.9 & 4 & 0.56 & 0.79 & 0.85 \\
\hline
\end{tabular}

CTRL = Control; $\mathrm{HCRN}=$ High Controlled Released Nutrients; LCRN = Low Controlled Released Nutrients;

$\mathrm{BDL}=$ Biodegradation Level; $\mathrm{TPH}=$ Total Petroleum Hydrocarbons; refer to text for diastereomer ratio definitions.

a - acquired quantitatively by GC-FID, ${ }^{\text {b }}$ - acquired by GC-MS 


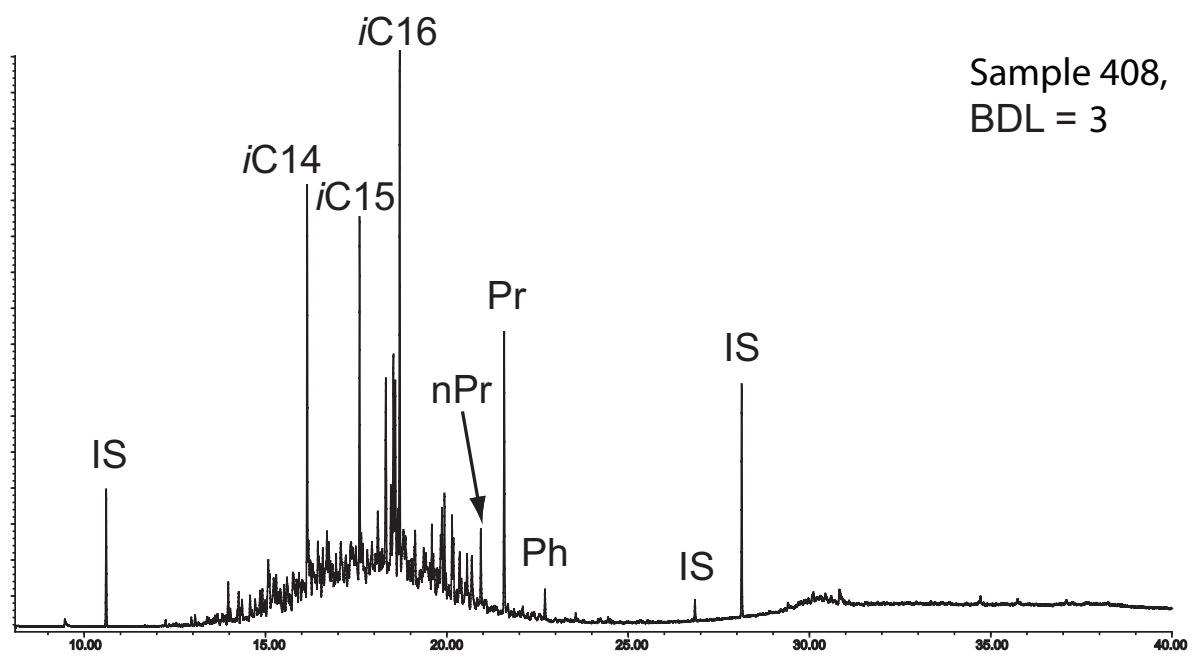

IS
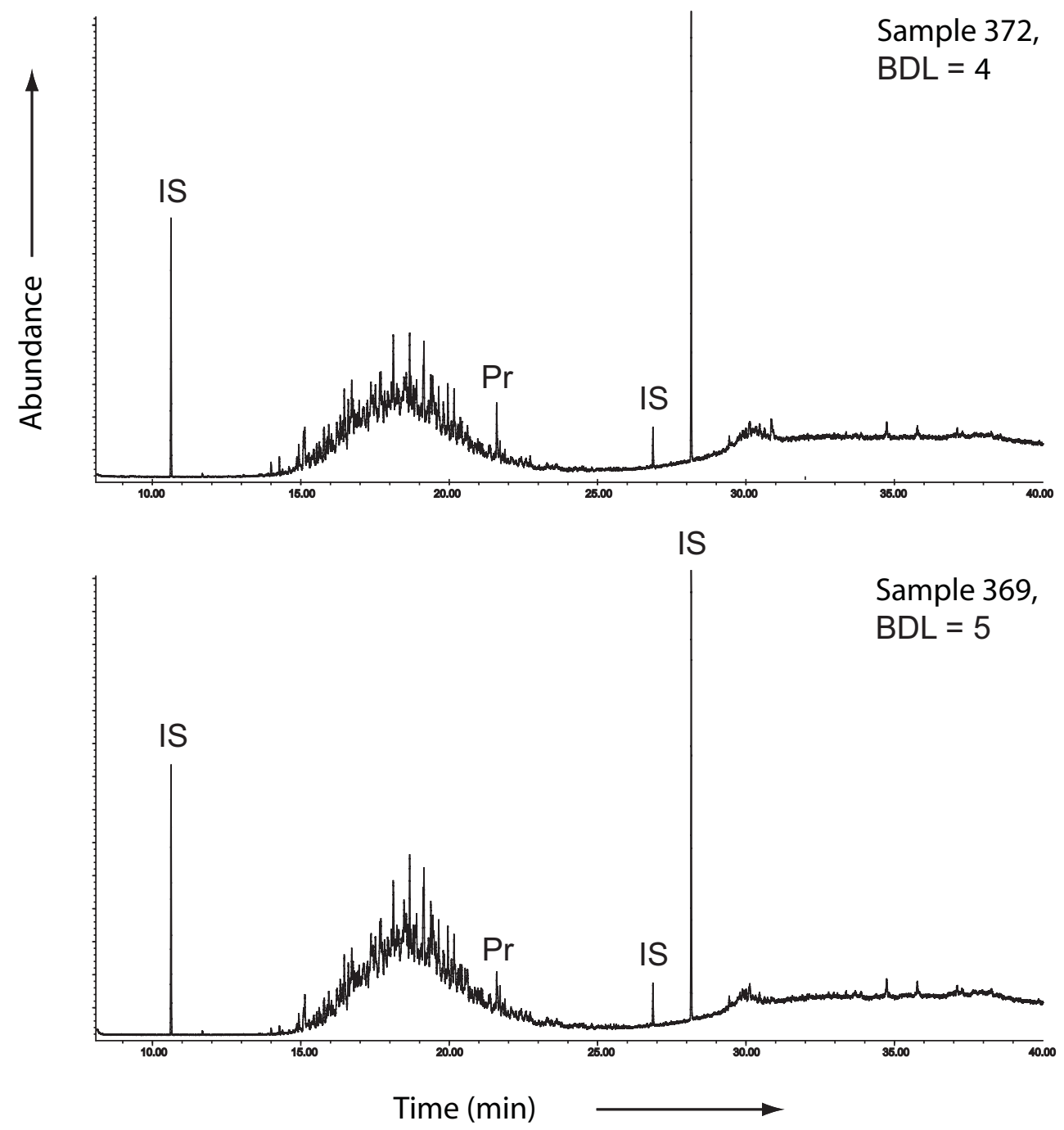

Figure S1. Full scan total ion chromatograms for the samples presented in Figure 2. Acquired using Method 1 and a scan range of $\mathrm{m} / \mathrm{z}$ 35-300 Da. IS denotes the internal standards (left to right) Cyclooctane, p-Terphenyl and 1-Bromoeicosane. Isoprenoids are denoted iC14-16, nPr (Norpristane), $\operatorname{Pr}$ (Pristane) and Ph (Phytane). 


\section{Pristane}

Phytane

\section{Biodegradation Level 3, Sample 408}
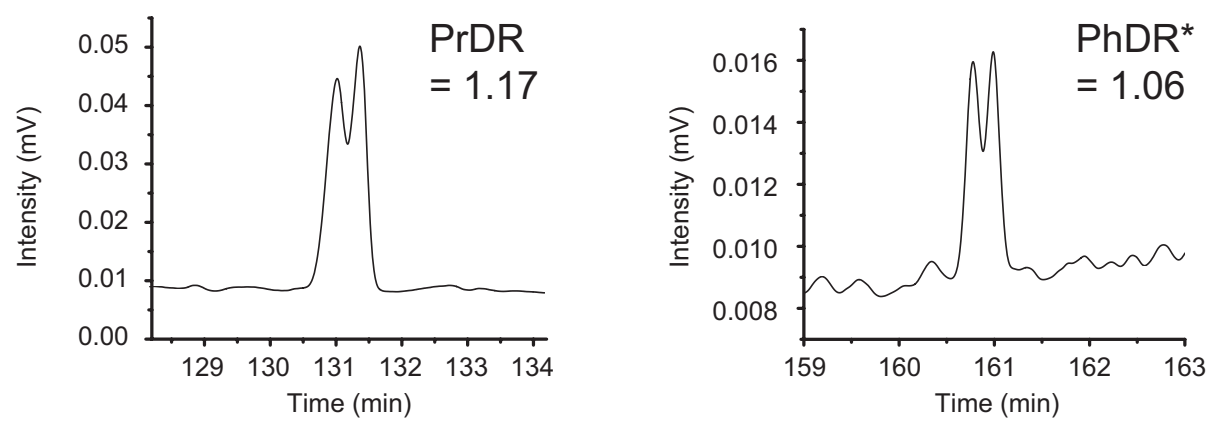

Biodegradation Level 4, Sample 372
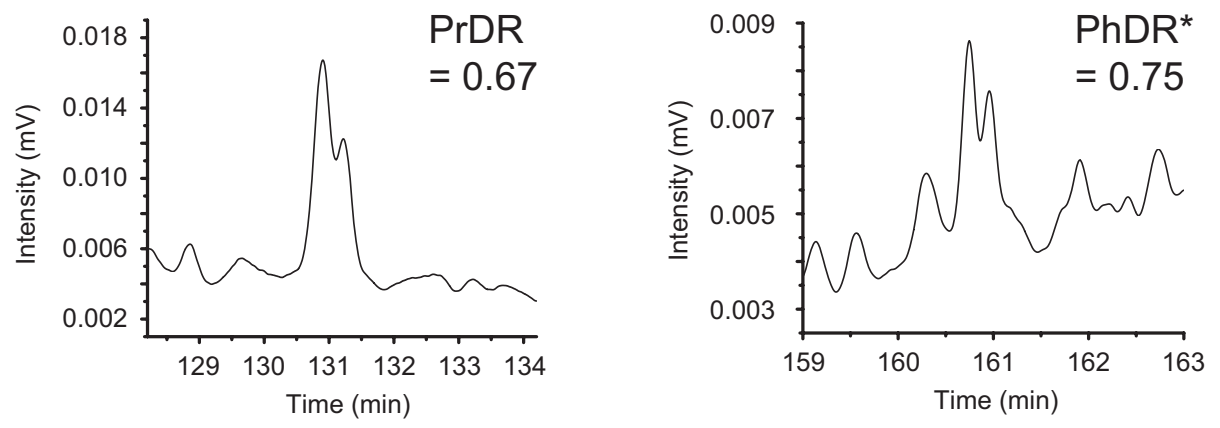

Biodegradation Level 5, Sample 369
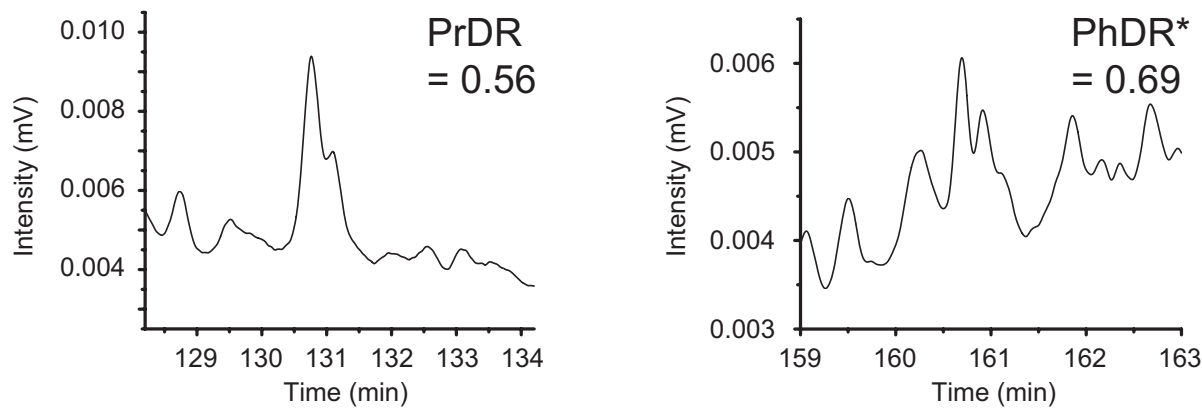

Figure S2. GC-FID chromatograms showing the variation of acyclic isoprenoid diastereomer ratios of pristane and phytane with aliphatic biodegradation level for three representative samples. Acquired on a Varian 3400 GC-FID using a constant column pressure of 25 psi Helium gas, an FID temperature of $350^{\circ} \mathrm{C}$ and a $1 \mathrm{uL}$ and a injection volume in splitless mode. 